\title{
Medial Femoral Trochlea Graft for Scaphoid Waist Nonunion: A Case Report and Review of the Literature
}

\author{
Bruno E. Crepaldi, MD ${ }^{1}$ Cameron Keating, MBBS, BMedSci ${ }^{1}$ Eugene T. Ek, MBBS, PhD ${ }^{2,3}$ \\ Stephen K. Y. Tham, MBBS $1,2,3$
}

${ }^{1}$ St. Vincent's Hand Surgery Unit, Victorian Hand Surgery Associates,

Fitzroy, Victoria, Australia

2 Hand and Wrist Biomechanics Laboratory, O’Brien Institute, Fitzroy, Victoria, Australia

${ }^{3}$ Dandenong Hospital Hand Unit, Dandenong, Victoria, Australia

\begin{abstract}
Address for correspondence Stephen K. Y. Tham, MBBS, Victorian Hand Surgery Associates, Suite C37, Level 3, Building C, St Vincent's Hospital, 41 Victoria Parade, Fitzroy 3065, VIC, Australia (e-mail: stham@bigpond.net.au).
\end{abstract}

J Wrist Surg 2020;9:186-189.

\begin{abstract}
Keywords

- scaphoid

- fracture

- carpal

- medial femoral trochlear

- non-union

The medial femoral trochlea (MFT) of the knee is a donor site for convex osteochondral vascularized bone that has been used for the salvage of fractures of the proximal pole of the scaphoid. Chronic nonunited fractures of the scaphoid may lead to a sequence of degenerative change often referred to as scaphoid nonunion advance collapse. The vascularized MFT osteochondral graft has been reported as a salvage procedure for fractures of the proximal pole of the scaphoid, in situations where fixation is not an option. In this "Special review," we describe the technique of free vascularized MFT graft in a case in which the nonunited scaphoid fracture was associated with segmental loss of the articular surface of the scaphoid waist. Given the likely progression of arthritis, if left untreated, we elected to treat this by replacing the lost articular surface using a vascularized intercalary osteochondral MFT graft between the nonunited scaphoid segments.
\end{abstract}

The free vascularized corticocancellous medial femoral condyle has been used for the treatment of scaphoid waist fracture nonunion. ${ }^{1}$ It is an intercalary bone graft placed between the proximal and distal scaphoid, after resection of the fracture nonunion, used in cases associated with avascular necrosis or recalcitrant non-union.

However, in fractures involving the proximal scaphoid associated with collapse of the proximal pole or fragmentation, the resected proximal pole can be treated by replacement with a free vascularized osteochondral graft from the medial femoral trochlea (MFT). ${ }^{2}$ This convex osteochondral segment of bone has been shown to have a similar arc of curvature to that of the proximal scaphoid, lunate, and capitate. ${ }^{3}$

The surgical treatment of nonunited fractures of the scaphoid waist is based on the principles of restoring anato-

received

November 26, 2018

accepted after revision

November 1, 2019

published online

December 17, 2019 my by correcting the deformity, providing stability, and is influenced by its viability and vascularity, osseous deficiency, and the cartilaginous status of the distal radius and scaphoid.

\section{Surgical Procedure}

A longitudinal incision was made dorsally at the junction of the third and fourth extensor compartments. The extensor pollicis longus tendon was released from its compartment and retracted radial wards. The scaphoid was exposed by a longitudinal capsulotomy. There was comminution of the scaphoid waist nonunion and an avascular proximal scaphoid evidenced by lack of punctate bleeding of the cancellous surface after curettage of fibrous tissue. The comminuted

Copyright @ 2020 by Thieme Medical Publishers, Inc., 333 Seventh Avenue, New York, NY 10001, USA.

DOI https://doi.org/ 10.1055/s-0039-3401015. ISSN 2163-3916. 
fragments were excised, and the fractured scaphoid ends trimmed with an oscillating saw leaving an osteochondral defect of $13 \mathrm{~mm}$ at the scaphoid waist between the proximal and distal scaphoid. A radial styloidectomy was performed for radial styloid arthritis.

Harvest of the MFT graft was performed from the ipsilateral knee as described by Bürger et al. ${ }^{2}$ The descending genicular vessels were identified, and an osteochondral graft was harvested measuring $13 \mathrm{~mm}$ in length and $10 \mathrm{~mm}$ in width ( - Fig. 1). It was transferred to the scaphoid defect and held with a $3.0 \mathrm{~mm}$ headless compression screw (Medartis,
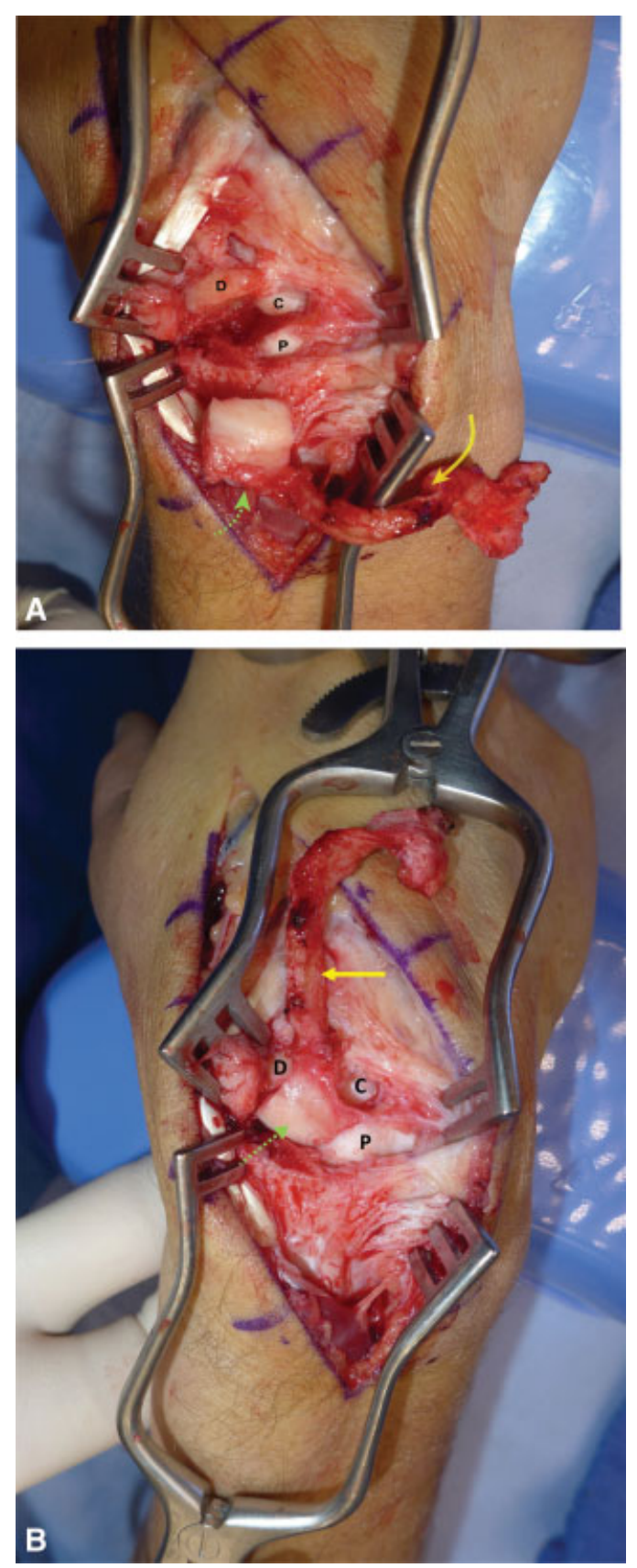

Fig. 1 (A) Osteochondral defect of $13 \mathrm{~mm}$ at the scaphoid waist; solid arrow-descending genicular vessels; dotted arrow-osteochondral graft; P-proximal pole of scaphoid; D-distal pole of scaphoid; Ccapitate. (B) After inset of medial femoral trochlear osteochondral graft to the waist of the scaphoid.
Basel, Switzerland). The screw position was confirmed with fluoroscopy. The descending genicular artery was repaired end-to-side to the radial artery at the anatomical snuffbox and two venae comitans were anastomosed to adjacent veins with 9/0 nylon suture. The wrist was immobilized in a long arm cast for 2 weeks and a thermoplastic wrist splint for 6 weeks.

\section{Case Presentation}

A 24-year-old male underwent internal fixation for a fractured right scaphoid. However, there was persistent nonunion despite repeat fixation with Kirschner wires and nonvascularized iliac crest bone graft (-Fig. 2 ).

Examination of his wrist showed tenderness in the region of the scaphoid waist with wrist flexion of 20 degrees, extension of 10 degrees, radial deviation of 0 degree, and ulnar deviation of 30 degrees. His grip strength was $21 \mathrm{~kg}$ on the affected side and $35 \mathrm{~kg}$ on the unaffected side. His patient rated wrist evaluation (PRWE) score was 59 and the disabilities of the arm, shoulder and hand (DASH) score was 49.

Plain radiographs (- Fig. $3 \mathbf{A}$ and B) and computed tomography scan ( - Fig. $\mathbf{3 C}$ and $\mathbf{D}$ ) revealed a segmental articular defect of the scaphoid waist and radial styloid arthritis.

Follow-up radiograph at 2 months (-Fig. 4A) showed healing of the fracture. At latest follow-up, 36 months after the procedure, his wrist flexion was 30 degrees, extension of 20 degrees, radial deviation at 10 degrees, and ulnar deviation at 30 degrees. His grip strength was $23 \mathrm{~kg}$. His PRWE score was 17 and his DASH score was 15 . There were no wrist symptoms. He complained of minor knee discomfort.

\section{Discussion}

Chronic nonunited fractures of the scaphoid may lead to a sequence of degenerative change often referred to as scaphoid nonunion advance collapse. ${ }^{4}$ These scaphoid fractures involving the waist may result in collapse of the palmar surface of the scaphoid, resulting in a humpback deformity and a nondissociative dorsal intercalated segment instability In this reported case, the nonunited scaphoid fracture was associated with segmental loss of the proximal articular surface of the scaphoid waist. This was most likely iatrogenic, although we do not have access to the initial radiographs from the referring surgeon. Given the likely progression of arthritis, if left untreated, at the articulation between the scaphoid and radius caused by its articular incongruity and altered biomechanics and subsequent midcarpal arthritis associated with dorsal intercalated instability from scaphoid collapse, we elected to treat this by replacing the lost articular surface with a vascularized intercalary osteochondral MFT graft between the nonunited scaphoid segments.

The use of vascularized osteochondral grafts from the distal femur holds promise because of its morphological similarity to the convex articular surfaces of the scaphoid, lunate, and capitate. ${ }^{3}$ The vascularized MFT osteochondral graft has been used as a salvage procedure, replacing the proximal pole of the scaphoid, in situations where fixation of 


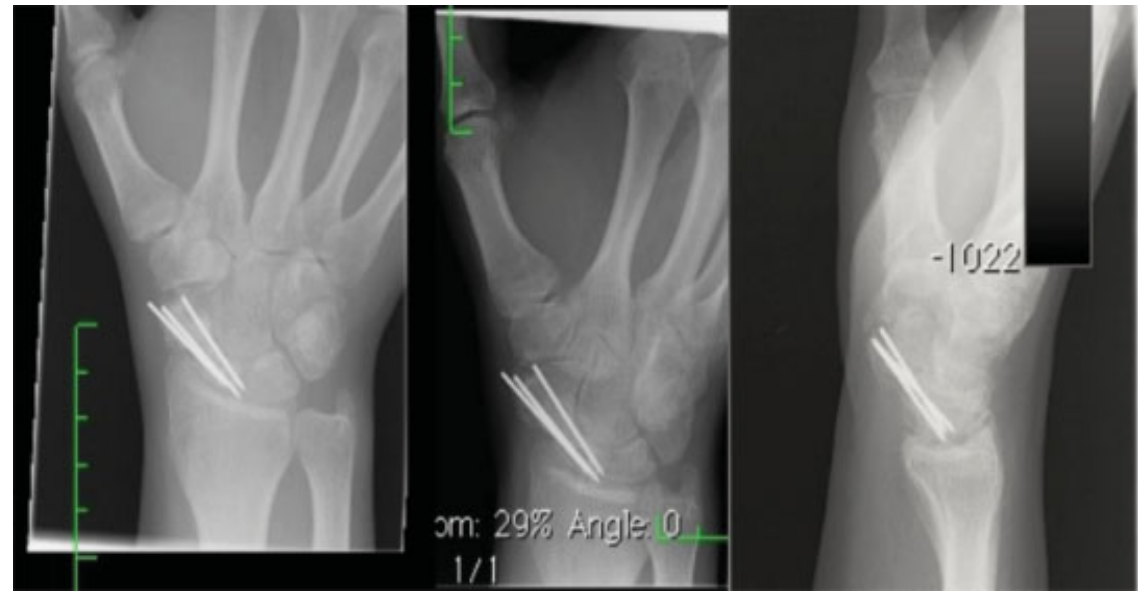

Fig. 2 Plain radiographs of scaphoid after iliac crest bone graft and Kirshner wire fixation.

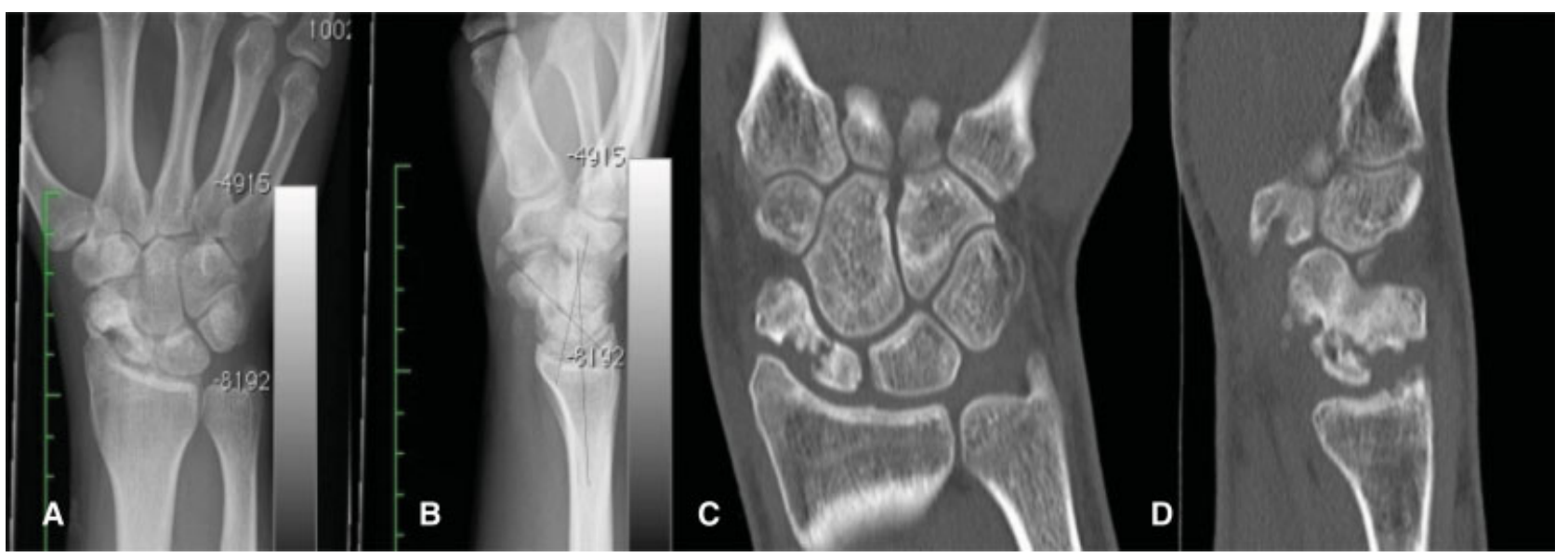

Fig. 3 Plain radiographs ( $A$ and $B$ ) and computed tomography images ( $C$ and $D$ ) show a nonunited scaphoid waist fracture with diffuse sclerotic changes of the proximal and distal poles, and deficient articular surface of the waist.

the scaphoid is not an option. The premise of replacing the proximal scaphoid with the MFT is that the outcome would be better and more durable than those obtained by salvage options such as scaphoid excision and four corner arthrode- sis or proximal row carpectomy, as this fractures often involve the young.

Bürger et $\mathrm{al}^{2}$ reported on 16 patients treated with MFT graft for scaphoid reconstruction. They reported healing in 15 of 16

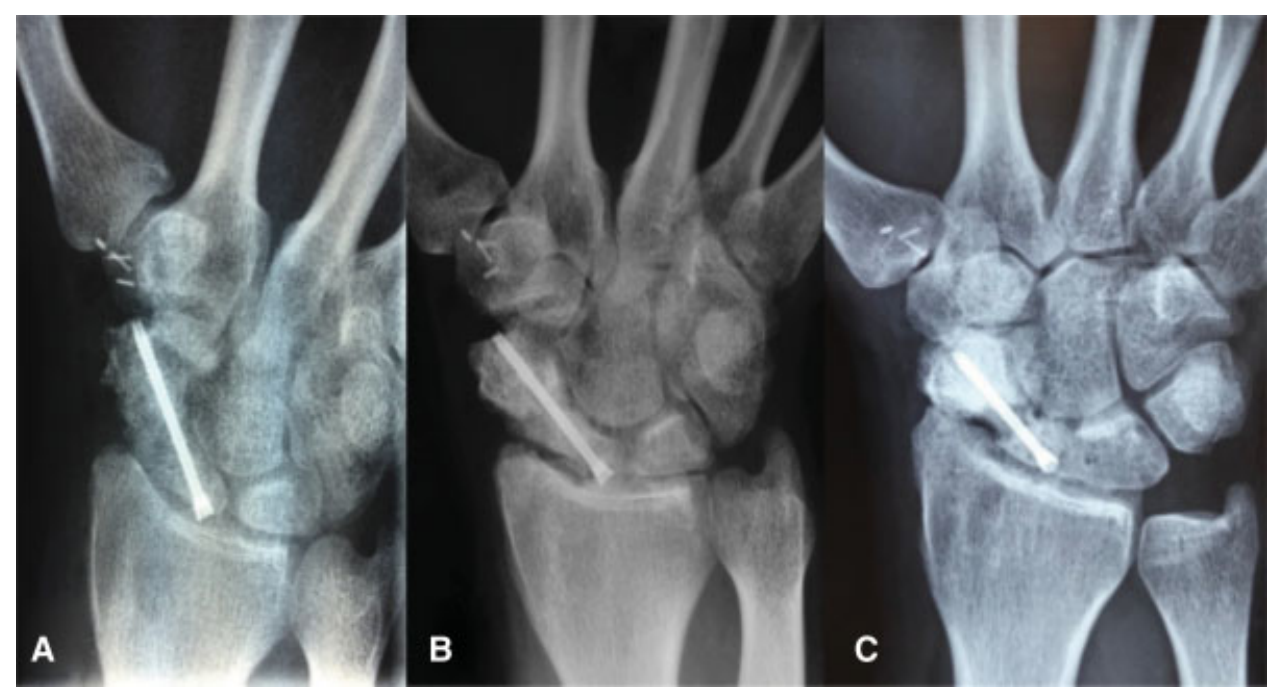

Fig. 4 Postoperative plain radiographs at (A) 2 months, (B) 12 months, and (C) 36 months. 
reconstructed scaphoids. One scaphoid failed to unite. The patient was a smoker and underwent additional surgery with revision screw fixation and bone graft. All patients experienced at least some pain relief, return of grip strength, and preservation of preoperative range of motion. At an average follow-up of 14 months, there were no arthritic changes and patients reported minimal or no pain at the knee donor site.

In 2014, Higgins and Bürger ${ }^{5}$ reported on patients who underwent MFT osteochondral reconstruction and included sixteen for scaphoid nonunion, sixteen for reconstruction of the lunate in Kienböck's disease, five capitate reconstructions, two lateral femoral trochlear reconstructions for medial tarsal avascular necrosis, and five posteromedial femoral osteochondral reconstructions of the humeral capitellum for posttraumatic arthritis. This resulted in osseous healing and restoration of joint alignment in all cases. The versatility and use of osteochondral flaps from the MFT, lateral femoral trochlear and proximal third metatarsal for the reconstruction of the articular surfaces of the scaphoid, lunate, capitate, and distal radius have been described. ${ }^{6}$

We have used the free vascularized MFT graft to replace the proximal scaphoid in 12 cases. The surgical approach is better performed from dorsal for ease of access to the scaphoid and recipient vessels, for preservation of the supporting palmar ligaments and for ease of fixation of the MFT graft to the distal scaphoid. Templating the defect with bone cement will minimize the size of the harvested cartilage from the knee. In cases involving the proximal scaphoid, correct templating is ensured by placing temporary Kirschner wires between the distal scaphoid and capitate after the scaphoid is extended to a normal radioscaphoid angle of between 30 and 60 degrees by ulnar deviation of the wrist. This must be performed prior to resection of the proximal scaphoid as the radioscaphoid angle cannot be measured after its resection. Templating of the defect is performed by using the cut tip of a surgical glove and filling it with polymethyl methacrylate (PMMA) bone cement. After the cement has slightly harden, the wrist is distracted and the cement-filled glove tip is inserted into the proximal scaphoid defect, making a template of the defect. We have not required the harvest of a skin paddle for skin coverage.

The potential consequence for the knee is of concern. However, Windhofer et $\mathrm{al}^{7}$ in a retrospective study of 57 patients who had undergone MFT graft harvest for scaphoid or lunate reconstruction, and with a mean follow-up of 27 months, reported that donor site discomfort was the most frequent complaint and that patients required $\sim 3$ months to return to preoperative level of comfort and use. In a minority of patients, persistent mild occasional or daily discomfort was reported at long-term follow-up. Despite these findings, their study demonstrated high patient satisfaction and willingness to undergo the same procedure.

Other motion-sparing surgical interventions that may be considered, particularly where degenerative disease of the wrist is associated with chronic scaphoid nonunion, are selective neurectomy, scaphoid excision with four-corner arthrodesis, or proximal row carpectomy. Four-corner arthrodesis and proximal row carpectomy are generally successful in relieving pain and preserving motion of the wrist though Wagner et al, ${ }^{8}$ suggested that proximal row carpectomy resulted in better range of motion and fewer complications in patients younger than 45 years. Though the reported range of motion after proximal row carpectomy or four-corner arthrodesis is greater than in our reported case, successful fixation of the scaphoid can mitigate the development of wrist arthritis. ${ }^{9}$

Placing an intercalary vascularized osteochondral graft to reconstitute a deficient scaphoid waist in this case re-established the scaphoid continuity, and potentially its kinematics. At 36 months, the patient wrist was asymptomatic, though there was persistence of wrist stiffness. Radiologically, there was evidence of resorption of the graft ( Fig. 4C) and it may indicate a degree of vascular compromise, though the graft appeared isodense compared with the adjacent carpal bones. No reference of this occurrence was noted by Bürger et al. ${ }^{2}$

Conflict of Interest

None declared.

\section{References}

1 Doi K, Oda T, Soo-Heong T, Nanda V. Free vascularized bone graft for nonunion of the scaphoid. J Hand Surg Am 2000;25(03): 507-519

2 Bürger HK, Windhofer C, Gaggl AJ, Higgins JP. Vascularized medial femoral trochlea osteocartilaginous flap reconstruction of proximal pole scaphoid nonunions. J Hand Surg Am 2013;38(04): 690-700

3 Hugon S, Koninckx A, Barbier O. Vascularized osteochondral graft from the medial femoral trochlea: anatomical study and clinical perspectives. Surg Radiol Anat 2010;32(09):817-825

4 Vender MI, Watson HK, Wiener BD, Black DM. Degenerative change in symptomatic scaphoid nonunion. J Hand Surg Am 1987;12(04):514-519

5 Higgins JP, Bürger HK. Osteochondral flaps from the distal femur: expanding applications, harvest sites, and indications. J Reconstr Microsurg 2014;30(07):483-490

6 Higgins JP, Bürger HK. The use of osteochondral flaps in the treatment of carpal disorders. J Hand Surg Eur Vol 2018;43(01): 48-56

7 Windhofer C, Wong VW, Larcher L, Paryavi E, Bürger HK, Higgins JP. Knee donor site morbidity following harvest of medial femoral trochlea osteochondral flaps for carpal reconstruction. J Hand Surg Am 2016;41(05):610-614.e1

8 Wagner ER, Werthel JD, Elhassan BT, Moran SL. Proximal row carpectomy and 4-corner arthrodesis in patients younger than age 45 years. J Hand Surg Am 2017;42(06):428-435

9 Düppe H, Johnell O, Lundborg G, Karlsson M, Redlund-Johnell I. Long-term results of fracture of the scaphoid. A follow-up study of more than thirty years. J Bone Joint Surg Am 1994;76(02): $249-252$ 